\section{Cureus}

\title{
High Speed Direct SAD Radiosurgery Beam Scanner
}

\author{
Walter Nikesch $^{1}$, James M. Hevezi ${ }^{2}$, Irene Monterroso ${ }^{3}$, Daniel Navarro ${ }^{4}$, James G. Schwade \\ 5
}

1. CyberKnife Center Of Palm Beach 2. Austin CyberKnife Center, Austin, TX 3. CyberKnife Center of Miami, Miami, FL 4. Advanced Radiation Measurements, Inc, 601 NE Emerson St., Port Saint Lucie, FL 34983 5. Cyberknife Center of Miami, University of Miami Miller School of Medicine

$\square$ Corresponding author: Walter Nikesch, wnikesch@cyberknifepalmbeach.com Disclosures can be found in Additional Information at the end of the article

\section{Abstract}

Background: Small field sizes used for stereotactic radiosurgery shouldn't require the use of large unwieldy three-dimensional water tanks for commissioning. A study was undertaken to determine whether a smaller volume of water generates significant scatter radiation to yield beam data measurements similar to those obtained using a large full-sized water tank. In addition, the use of a light, small volume tank, would allow for movement of the whole tank in space so that direct SAD beam measurements can be obtained in a much shorter time.

Methods: A novel new idea in scanner design was developed where a relatively small volume of water is moved in 3D space, keeping the detector centered in the tank in the horizontal X,Y plane and allowing for detector motion in the vertical Z direction. A separate vertical drive in the tank can move the probe detector independently of the external 3D (X,Y,Z) drives. This separate vertical $\mathrm{Z}$ drive is synchronized with the external $\mathrm{Z}$ drive to keep the detector at a fixed, constant distance from the linac radiation source (constant SAD). For TPR data collection, the tank vertical drive moves the detector down at the same speed as the external drive raises the tank yielding a direct TPR measurement. For radial, transverse, and diagonal profiles, the whole tank is moved across beam to accumulate cross profiles at various depths determined by the vertical drive in the tank. For percent depth dose measurements, the external drives remain stationary and only the in tank drive is used to move the detector down.

Results: The final design (20 cm cylinder, $40 \mathrm{~cm}$ height) required three gallons of water weighing about $24 \mathrm{lbs}$. A robust support system and strong drive motors were required to negate gravity effects. TPR measurements obtained in the down and up directions were compared and found to be nearly identical below depth of maximum build-up. TPR's, cross profiles at five depths, and percent depth doses were measured and compared to those obtained in a large tank. In all cases, the results were nearly identical. The greatest variation found was for the $6 \mathrm{~cm}$ field size profiles at $300 \mathrm{~mm}$ depth where the small tank showed values in the toe region, outside the primary beam, of about $0.5 \%$ less. In addition to direct SAD measurements, one of the greatest benefits of this system is the fast time of data acquisition. The total scanning time for one cone to obtain TPR and 10 cross profiles at five depths was under 10 minutes at the slowest, highest resolution, settings. For comparison, direct 12 point TPR measurements and SAD cross profile measurements using a standard large tank required significant operator interaction and took almost one hour to complete one cone.

Conclusions: A small volume scanning system was found to yield beam scanning results almost identical to those obtained in a large tank. In addition, direct SAD TPR and profile beam data for 12 cones and 12 IRIS field sizes can be obtained in less than one day. 
Categories: Medical Physics, Radiation Oncology, Miscellaneous

Keywords: treatment planning, data acquisition, beam scanner, commissioning, sad scanner, small beam measurements, srs, tpr measurement

\section{Introduction}

Radiation dosimetry calculations and treatment planning require information on the threedimensional intensity and distribution of radiation produced by medical linear accelerators. To obtain this data, precise measurements are performed using a radiation beam scanner (water phantom). The traditional 3D radiation beam scanner consists of a large water tank and a motorized three-dimensional positioning system which moves a radiation detector throughout the tank. The primary motions of the detector are vertical to measure percent depth dose or horizontal across the tank to measure beam profiles. A fixed reference detector is used to correct for accelerator anomalies by calculating a ratio of the signal detector output to the reference detector output. This system is capable of measuring beams as large as $40 \mathrm{x} 40 \mathrm{~cm}$ square. Data acquired by these systems is at a fixed radiation source to water surface distance (SSD). The basic design of the water phantom has not changed much since it was introduced over 30 years ago. However, during this time there have been major advances in radiation treatment delivery systems and dose calculation/treatment planning systems. One of these advancements has been in the development of dedicated treatment systems for radiosurgery and stereotactic body radiation therapy and their corresponding treatment planning systems. These devices tend to use radiation beams of much smaller size than traditional medical linear accelerators. For example, the range in field sizes in the CyberKnife is from $5 \mathrm{~mm}$ to $60 \mathrm{~mm}$. The treatment planning systems for many of these modern devices, including the CyberKnife, require beam data in a constant source detector-axis-distance (SAD) format instead of the traditional SSD format [1]. This data can be obtained from measurements obtained using traditional water phantoms in the SSD format using calculations derived from first principles using a simple geometric model. However, the results can vary depending on whether field size and peak scatter factor are defined at the surface or at depth and then applying a conversion factor [2]. Because of potential errors in the percent depth dose to TPR conversion, it is recommended that TPR's be measured directly. A method to directly measure TPR's was described several decades ago where the ionization chamber was held fixed at isocenter using an arm secured to the linac head [3]. The chamber is immersed in a water phantom supported on the treatment table. The water phantom is moved up and down by raising and lowering the treatment couch under computer control. This method works for measuring TPR's directly, but it has no ability to measure beam profiles. In addition, the vertical table drives and controls tend not to be as accurate and precise as those found on precision water phantoms.

Large water tanks are unwieldy for small beam measurements, and smaller water tanks may be more efficient to use in these instances, as long as the full scatter conditions can be attained with them. In addition, a scanning system that can accumulate the data directly with the precision necessary for these treatment techniques, quickly and in a SAD format, for direct input to the treatment planning systems used in these procedures would improve the quality of the data (no calculations necessary for SSD to SAD conversion) and significantly decrease the time required for beam commissioning. To that end, we describe a new water phantom scanner design (Advanced Radiation Measurements, Inc. Port St. Lucie, Fla) produced especially for the small fields used in radiosurgery applications. Attention in this design is placed on direct, precision measurements for the increased accuracy necessary for the small beams (as small as 5 $\mathrm{mm}$ ) used in these applications. Additionally, functionality is placed on obtaining all of the beam data necessary in scanning cross-profiles (including diagonal profiles) as well as obtaining depth and TPR profiles directly in a smooth scanning process. For these small beams, precision movement of the detector (usually a small point measuring diode) is paramount in 


\section{Cureus}

obtaining accurate data for radiosurgery applications.

\section{Materials And Methods}

A novel new idea in scanner design was developed where a radiation diode detector centered in a relatively small volume of water is moved in 3D space. This motion required the traditional $X$, $\mathrm{Y}$, and $\mathrm{Z}$ motor drives to move the tank and detector as one unit in space. An additional fourth motor drive was used for separate vertical detector only motion within the tank. This separate vertical $\mathrm{Z}$ drive is synchronized with the external $\mathrm{Z}$ drive to keep the detector at a fixed, constant distance from the linac radiation source (constant SAD). For TPR data collection, the tank vertical drive moves the detector down at the same speed as the external drive raises the tank yielding a direct TPR measurement. For beam profiles, the in tank $\mathrm{Z}$ drives move the detector to the desired depth and the external $\mathrm{Z}$ drive moves the tank up or down so the detector is at the correct SAD. The entire tank/detector assembly is then moved across the beam to obtain the desired profile. The scanner design took several iterations to arrive at the required precision and full-scatter requirements necessary. To investigate this scanning concept, the original prototype tank was square in cross section $(10 \times 10 \mathrm{~cm})$ with a $40 \mathrm{~cm}$ height. While this tank was very light, proving easy to move in space, it only provided full-scatter for the smallest field sizes. TPR and profile data for the small field sizes matched CyberKnife composite beam data quite well, proving that this scanning concept was a viable idea. The next water tank design was cylindrical $40 \mathrm{~cm}$ in height and the diameter was $5 \mathrm{~cm}$ larger all around than the largest diameter cone $(6 \mathrm{~cm})$ which resulted in a $16 \mathrm{~cm}$ (excluding Lucite walls) diameter cylinder. This tank required two gallons of water to fill and weighed approximately 17 pounds. To move this weight required stronger motors than those used in traditional water tanks. TPR data acquired with this cylinder matched composite data quite well; however, profiles at depths over $200 \mathrm{~mm}$ for the largest $(6 \mathrm{~cm})$ field size did not match perfectly, suggesting additional scatter was required. The final tank design increased the tank diameter to $20 \mathrm{~cm}$. This required almost three gallons of water to fill. The additional weight ( $24 \mathrm{lbs}$ total) required even stronger motors to move the assembly with the desired precision. Figure $1 a$ shows the final scanner configuration and Figure $1 b$ shows just the tank with its separate vertical $\mathrm{Z}$ drive. Using this system, an entire beam data set was acquired for the CyberKnife system.

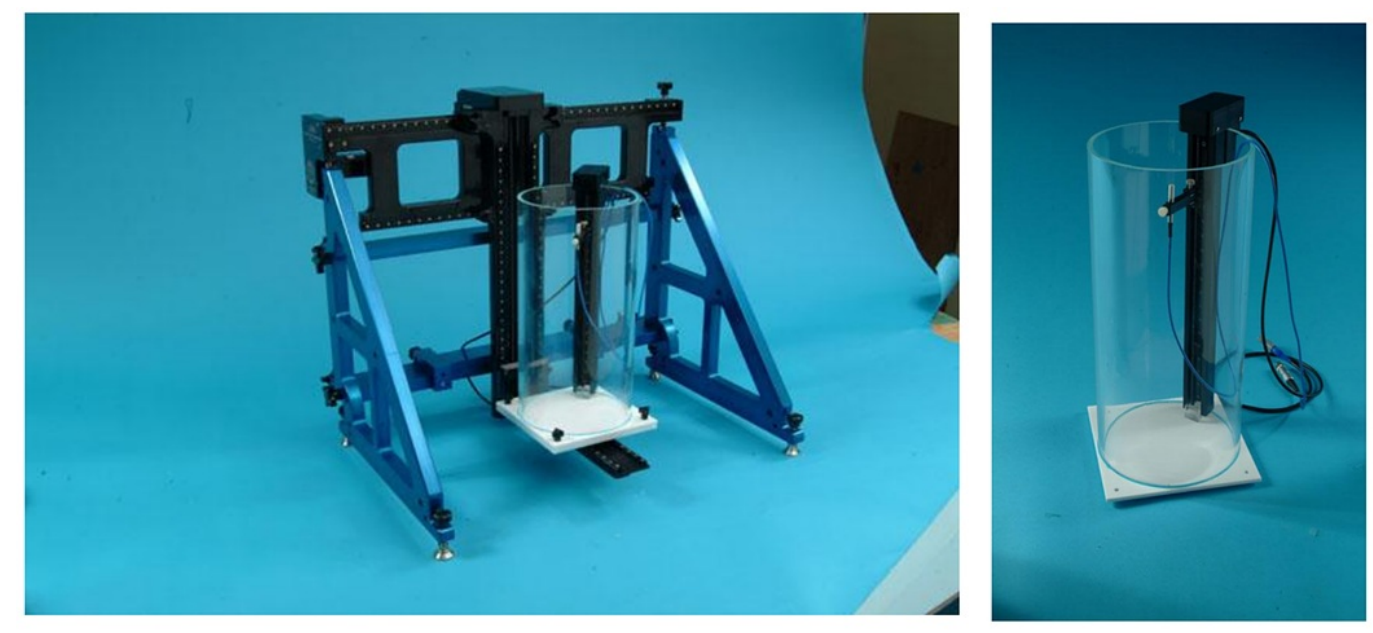

FIGURE 1: Equipment

1A: SAD scanner assembly with $20 \mathrm{~cm}$ diameter tank $1 \mathrm{~B}: 20 \mathrm{~cm}$ diameter tank showing separate internal $Z$ axis positioning drive holding the diode detector 


\section{Cureus}

The largest field sizes (40 mm, $50 \mathrm{~mm}$ and $60 \mathrm{~mm}$ ) were then re-measured using a traditional large water tank. It should be noted that for this comparison the same motorized scanning system was used. The cylindrical tank with its radial support were removed (Figure $2 a$ ). A new radial arm which only holds the diode was added and the entire X, Y, and Z motor assembly was placed on top of a large traditional water tank (Figure $2 \mathrm{~b}$ ). Also for accurate comparison, the data measured with the large tank was in SAD format. TPR's were measured each point separately by pushing in the bird cage into the water phantom ("bird cage technique"). The Accuray-recommended 12 depth points were measured. Also, each large tank profile was measured separately at $80 \mathrm{~cm}$ SAD. The $15 \mathrm{~mm}$ profile was measured at $78.5 \mathrm{SSD}$. The robot's vertical position was then lowered a distance similar to the increase in depth of the next profile and so forth until the $300 \mathrm{~mm}$ profile was measured.
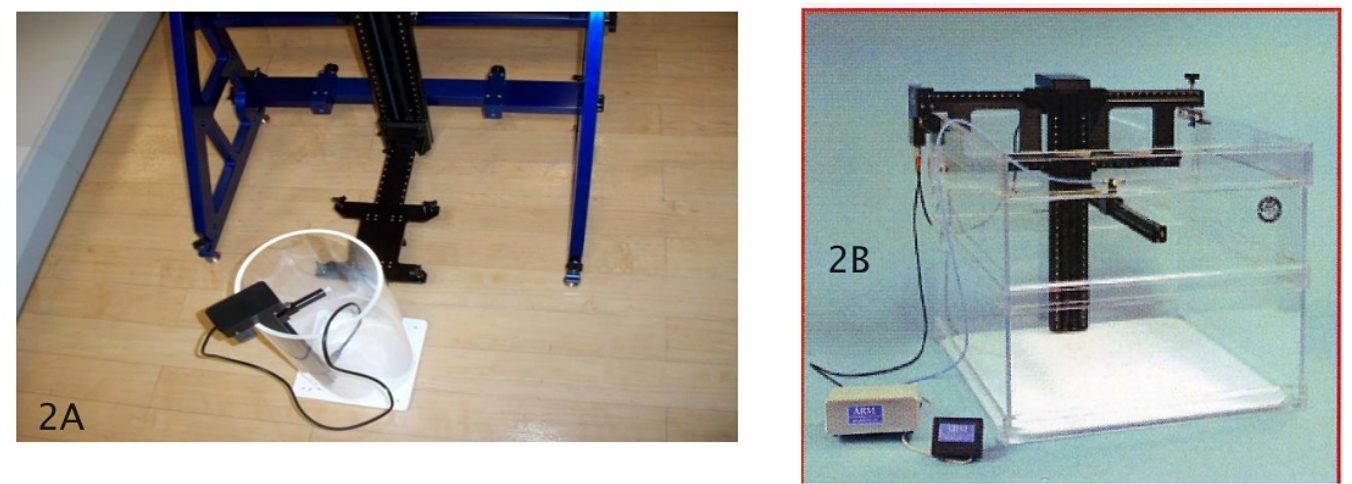

\section{FIGURE 2: Equipment}

2A: $20 \mathrm{~cm}$ tank and support arm easily removed $2 \mathrm{~B}$ : Large tank used for comparison. This tank used the same $X, Y$, and $Z$ drive motors and assemblies used to position the $20 \mathrm{~cm}$ diameter tank.

\section{Results}

Our initial concern was a loss in precision due to the weight of the tank/detector assembly (24 lbs), so that the cylindrical tank moves accurately, strong motors and a robust support system is required. This support system is important for accurate cross profile scans, especially when scans are performed in the inboard to outboard directions because a "diving board" effect could affect cross profiles in a detrimental manner as the tank is moved in the outboard direction. Measurements made with a precision level shows a maximum 0.10 "diving board" deflection as the tank is moved from its most inboard to most outboard position. This deflection is about $0.3 \mathrm{~mm}$ for a $160 \mathrm{~mm}$ outboard motion which was felt to be insignificant. Our next concern was the effect of gravity. TPR data was obtained with the detector starting at the water surface and ending at $300 \mathrm{~mm}$ depth (tank moving up against gravity). This was compared to TPR data obtained in the opposite direction starting at depth $300 \mathrm{~mm}$ and ending at the water surface (tank moving down with gravity). The differences noted between these two scanning motions were minimal with all differences past maximum build-up depth within $1 \%$. TPR data obtained with the tank moving with and against gravity are quite similar, showing minimal gravity effect. Larger differences were noted in the build-up region were most likely due to water tension effects at the surface. In either case, all further TPR data presented here is the average of two scans obtained with and against gravity.

While an entire beam data set was obtained for all 12 cones using the new scanning system, comparison of this data to data obtained with a traditional large water tank and presented here 


\section{Cureus}

is mostly for the $60 \mathrm{~mm}$ cone size. It was felt that if data for the largest field size compared well, then this tank supplies sufficient scatter and the smaller field sizes will also compare well. Large and small tank TPR data for the $60 \mathrm{~mm}$ cone is presented in Table 1.

\section{TPR Small Tank}

Depth $\mathrm{mm}$

5

10

15

20

30

50

100

150

200

250

300
$60 \mathrm{~mm}$

0.85

0.971

1

0.994

0.966

0.897

0.72

0.568

0.451

0.357

0.285

\section{TPR Large Tank}

$60 \mathrm{~mm} \quad \%$ Diff

0.872

$2.20 \%$

0.977

$0.50 \%$

$0.00 \%$

0.995

$0.10 \%$

0.965

$-0.10 \%$

0.894

$-0.30 \%$

0.716

$-0.40 \%$

0.566

$-0.20 \%$

0.448

$-0.30 \%$

0.356

$-0.10 \%$

0.284

$-0.10 \%$

TABLE 1: TPR values obtained with the $20 \mathrm{~cm}$ diameter tank (Small Tank) and obtained using a traditional large water tank (Large Tank). The large tank values were direct point by point measurements.

Percent depth dose data can also be obtained with the new scanning system. In this case, the tank is not moved and just the radiation diode detector is moved in the vertical direction as with traditional scanners. In this case, the field size would increase with depth, starting at 60 $\mathrm{mm}$ at the surface and ending at about $83 \mathrm{~mm}$ at depth $300 \mathrm{~mm}$. Accurate measurements at larger field sizes would require more scatter volume. To verify whether there is enough scatter volume, percent depth dose data was acquired with both the new small tank and a traditional large tank. The results are shown in Table 2. 


\section{Cureus}

\begin{tabular}{|c|c|c|c|c|}
\hline Depth mm & Field Size mm & \%DD Small Tank & \%DD Large Tank & $\%$ Diff \\
\hline 10 & 60.8 & 98.3 & 98.1 & $0.40 \%$ \\
\hline 15 & 61.1 & 100 & $10000.00 \%$ & $0.00 \%$ \\
\hline 20 & 61.5 & 98.7 & $9850.00 \%$ & $-0.20 \%$ \\
\hline 30 & 62.3 & 93.6 & $9330.00 \%$ & $-0.30 \%$ \\
\hline 50 & 63.8 & 82.9 & $8290.00 \%$ & $0.00 \%$ \\
\hline 100 & 67.5 & 59.1 & $5960.00 \%$ & $-0.10 \%$ \\
\hline 150 & 71.3 & 42.8 & $4310.00 \%$ & $0.30 \%$ \\
\hline 200 & 75 & 30.9 & $3100.00 \%$ & $0.10 \%$ \\
\hline 250 & 78.8 & 22.4 & $2260.00 \%$ & $0.20 \%$ \\
\hline 300 & 82.5 & 16.5 & $1670.00 \%$ & $0.20 \%$ \\
\hline
\end{tabular}

TABLE 2: Percent depth dose values obtained with the $20 \mathrm{~cm}$ water tank (Small Tank) and the traditional water tank (Large Tank)

The results show that the small tank provides sufficient scatter, even at greatest depths. Cross beam profiles were obtained at depths required for Multi-Plan commissioning, $15 \mathrm{~mm}, 50$ $\mathrm{mm}, 100 \mathrm{~mm}, 200 \mathrm{~mm}$, and $300 \mathrm{~mm}$. Again, profile data was obtained using both the small 20 cm cylindrical tank and the traditional large water tank. A comparison of the penumbra widths for both tanks using the $60 \mathrm{~mm}$ cone is given in Table 3. The penumbra width was defined as the distance between the $80 \%$ and $20 \%$ isodose values. The penumbra results for both tanks were found to be within $0.1 \mathrm{~mm}$. 


\section{Cureus}

$60 \mathrm{~mm}$ cone penumbra width $(80 \%-20 \%)$

$\begin{array}{llc}\text { Depth } & \text { Small Tank } & \text { Large Tank } \\ 15 \mathrm{~mm} & 3.7 \mathrm{~mm} & 3.7 \mathrm{~mm} \\ 50 \mathrm{~mm} & 4.4 \mathrm{~mm} & 4.4 \mathrm{~mm} \\ 100 \mathrm{~mm} & 5.0 \mathrm{~mm} & 4.9 \mathrm{~mm} \\ 200 \mathrm{~mm} & 5.4 \mathrm{~mm} & 5.5 \mathrm{~mm} \\ 300 \mathrm{~mm} & 5.8 \mathrm{~mm} & 5.9 \mathrm{~mm}\end{array}$

TABLE 3: Penumbra values obtained with the $20 \mathrm{~cm}$ water tank (Small Tank) and the traditional water tank (Large Tank)

Full cross beam profiles were compared at the five depths for both tanks. Profile plots were superimposed and no differences between tanks were noted for the $60 \mathrm{~mm}$ cone at depths 15 $\mathrm{mm}, 50 \mathrm{~mm}$, and $100 \mathrm{~mm}$. At $200 \mathrm{~mm}$ and $300 \mathrm{~mm}$, differences were noted in the "toe" region of the profiles. At $200 \mathrm{~mm}$, the values were approximately $0.3 \%$ higher (relative to the CAX value), and at $300 \mathrm{~mm}$, the toe region was about $0.5 \%$ higher. These profiles are shown in Figures 3 and 4.

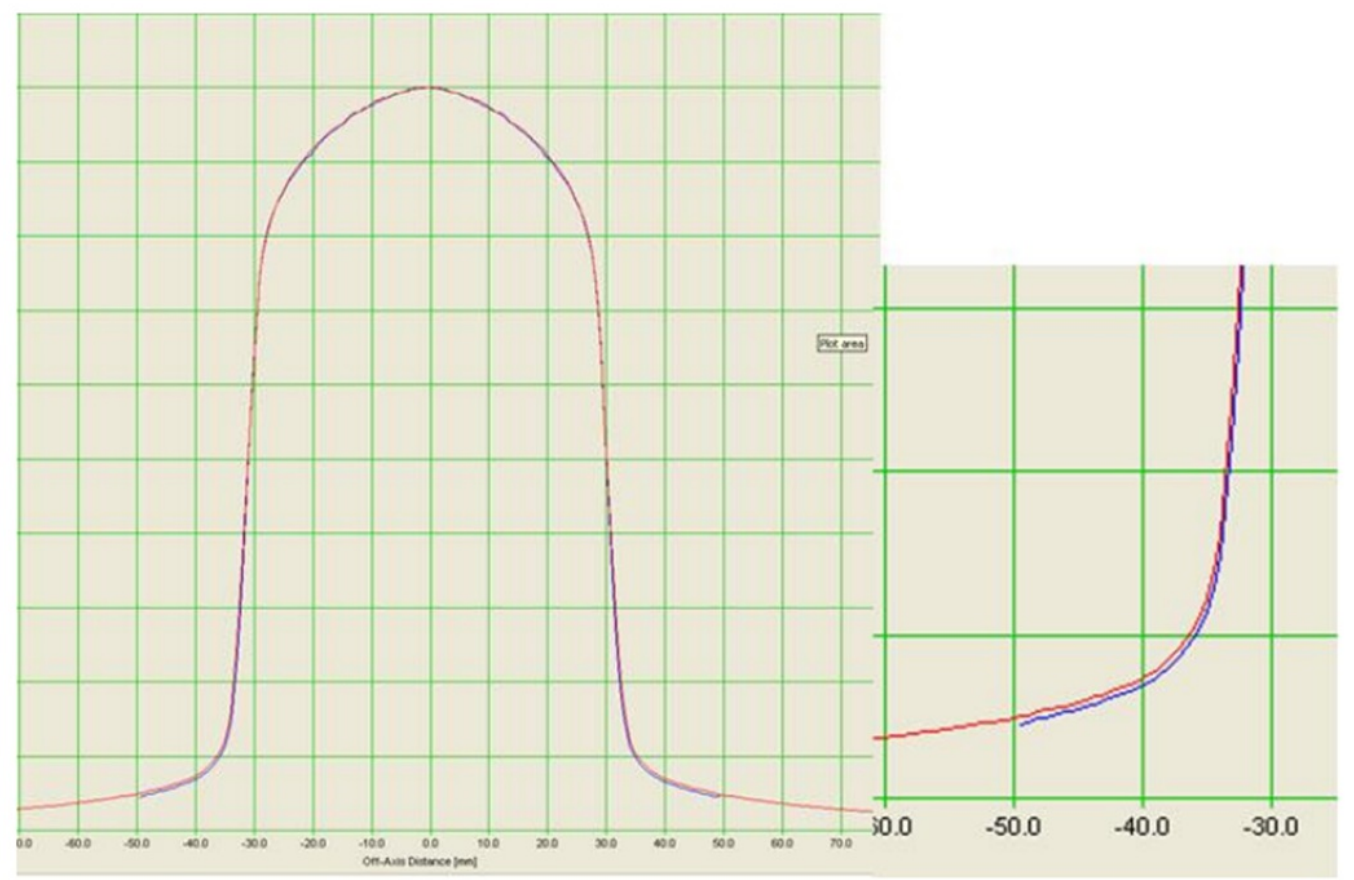

FIGURE 3: $200 \mathrm{~mm}$ depth profile for the $60 \mathrm{~mm}$ cone

$200 \mathrm{~mm}$ depth profile for the $60 \mathrm{~mm}$ cone. Readings obtained using the small tank were $0.3 \%$ lower in the toe region than those obtained using the large tank. 


\section{Cureus}

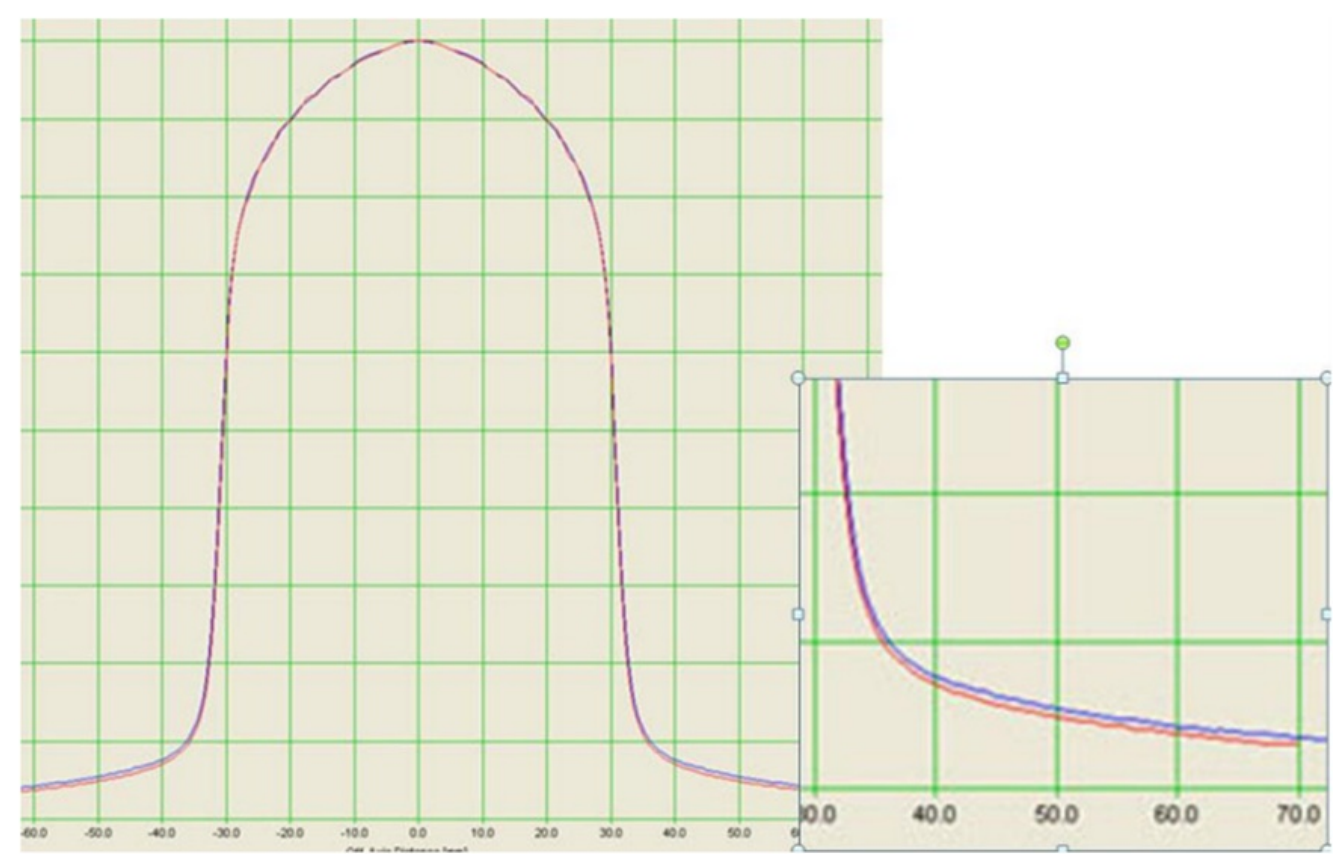

FIGURE 4: $300 \mathrm{~mm}$ depth profile for the $60 \mathrm{~mm}$ cone

$300 \mathrm{~mm}$ depth profile for the $60 \mathrm{~mm}$ cone. Readings obtained using the small tank were $0.5 \%$ lower in the toe region than those obtained using the large tank.

Profile sections in the toe region for all five depths for the $50 \mathrm{~mm}$ cone size and $40 \mathrm{~mm}$ cone size are shown in Figures 5 and 6, respectively. For the $50 \mathrm{~mm}$ cone, all profiles overlaid nicely except the $300 \mathrm{~mm}$ depth profile which showed a difference of about $0.3 \%$. There were no differences noted in any profiles for the $40 \mathrm{~mm}$ cone. 


\section{Cureus}

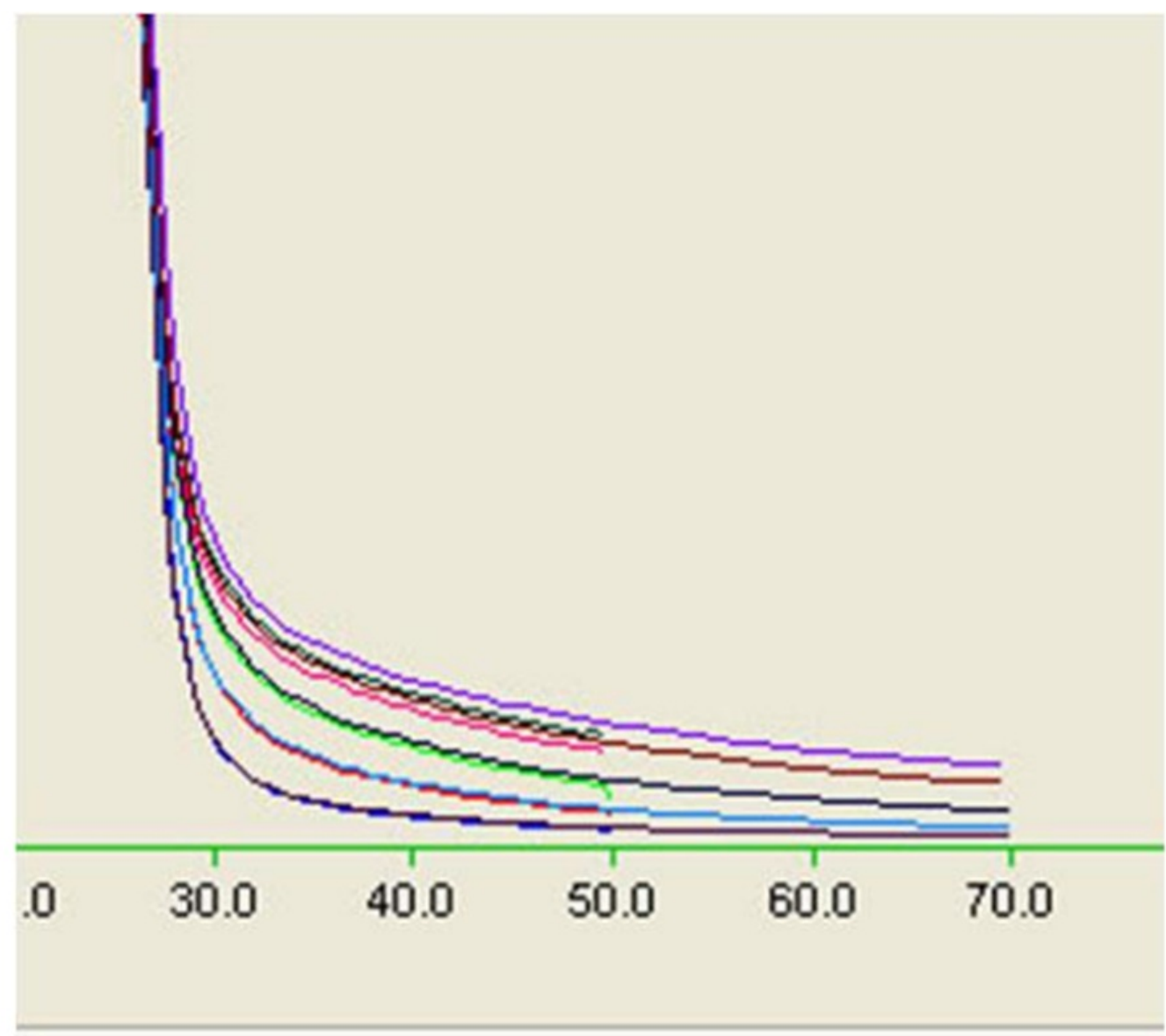

FIGURE 5: $50 \mathrm{~mm}$ cone profile comparison

$50 \mathrm{~mm}$ cone profile comparison for the toe region (note small tank measurements stopped at 50 $\mathrm{mm}$ off axis). The profiles shown were (lowest to highest) at depths $15 \mathrm{~mm}, 50 \mathrm{~mm}, 100 \mathrm{~mm}$, $200 \mathrm{~mm}$, and $300 \mathrm{~mm}$. A $0.3 \%$ lower small tank reading noted at $300 \mathrm{~mm}$ 


\section{Cureus}

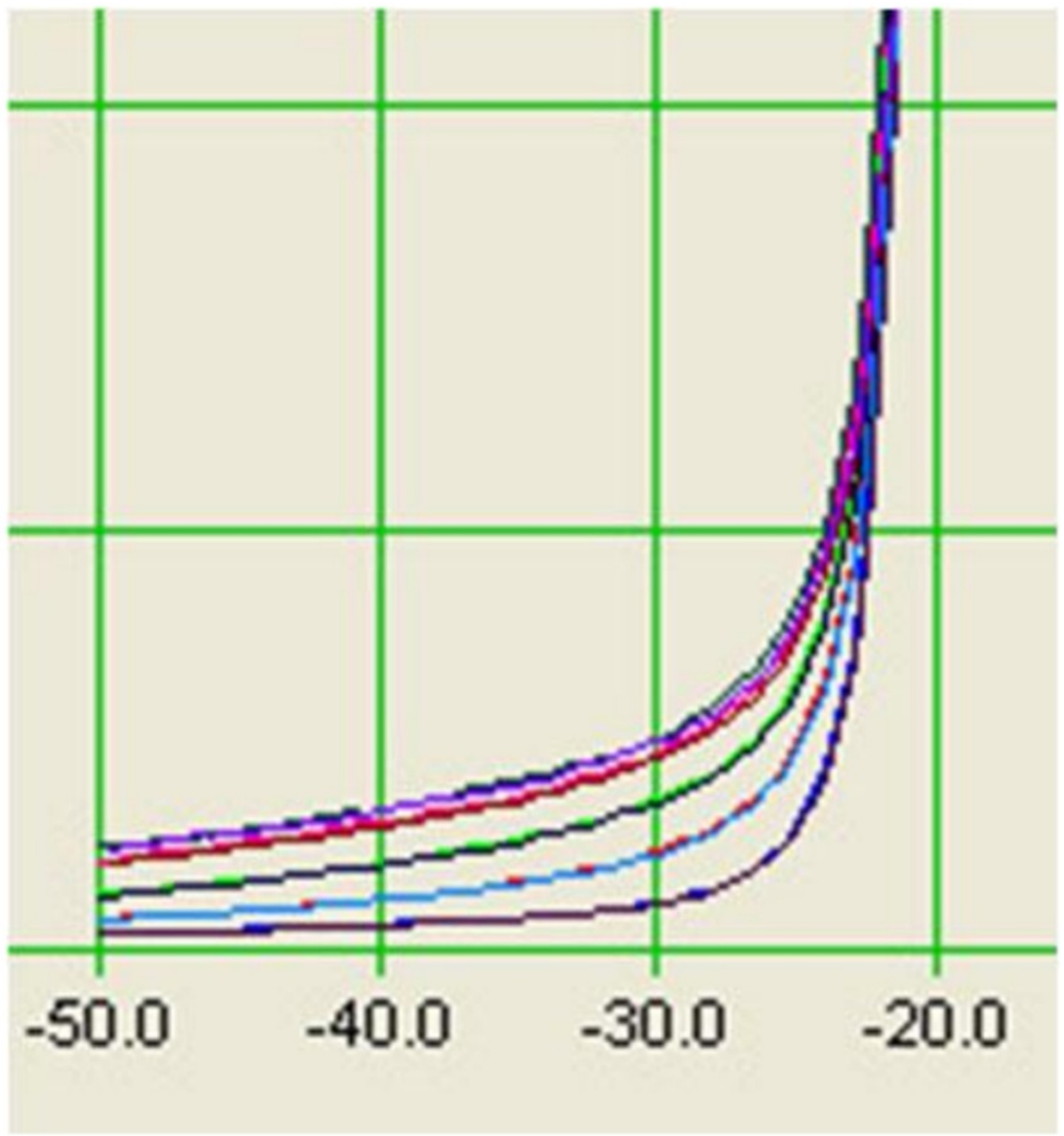

\section{FIGURE 6: $40 \mathrm{~mm}$ cone profile comparison}

$40 \mathrm{~mm}$ cone profile comparison of the toe region showing the same five profile depths as in Figure 5. Overlays of small tank and large tank profiles shows a perfect match.

\section{Discussion}

CyberKnife beam depth and profile data were obtained using a new small scanner which rapidly obtains the data in a SAD format. This data was compared to that obtained using a traditional large water tank. All depth data (TPR) was found to be identical within experimental set-up between the two systems. The profile data was also found to be identical, except for the largest two cones. For the $50 \mathrm{~mm}$ cone at depth $300 \mathrm{~mm}$, the toe region was found to be about $0.3 \%$ higher for the larger tank. For the $60 \mathrm{~mm}$ cone, the toe region readings were found to be about $0.3 \%$ higher at $200 \mathrm{~mm}$ and $0.5 \%$ higher at depth $300 \mathrm{~mm}$ using the larger tank. All other measurements for all smaller cones were found to be identical within experimental error. The slightly lower measurements at greatest depths for the largest cones were most likely the result of less scatter generated in the smaller tank. We feel that these differences are clinically insignificant since the differences are very small and only occur at large depths at the largest cone sizes. 
In addition, actual patients are much smaller than the large water tank and probably generate scatter closer to what's generated by the $20 \mathrm{~cm}$ cylinder. Therefore, this data should be acceptable for clinical beam commissioning. In addition, it should be noted that the entire data set for the $60 \mathrm{~mm}$ cone (TPR, five transverse profiles, and five radial profiles) was obtained in about 10 minutes. Depth points were obtained every $1 \mathrm{~mm}$ and profile points every $0.5 \mathrm{~mm}$. For cones $25 \mathrm{~mm}$ and smaller, the profile data separation was reduced to $0.2 \mathrm{~mm}$, so these cones took a couple of minutes longer. In either case, the entire data set for 12 cones and 12 IRIS field sizes, which includes two profiles sets, the 2 nd set at 150 off the 1 st set, can easily be obtained in one day. SAD data obtained using the traditional large tank with the "bird cage" technique and measuring 12 points separately takes about 30 minutes for each cone and IRIS field size. Likewise, SAD profile measurements take additional time since the robot vertical position needs to be moved for each depth. Taking SAD measurements with a large tank is cumbersome and prone to mistakes. These measurements routinely take a few days to a week, depending on experience.

\section{Conclusions}

A small volume scanning system was found to yield beam scanning results almost identical to those obtained in a large tank. Variations due to lack of sufficient scatter were very small and only noted at the largest depths for the largest field sizes. In addition, direct SAD TPR and profile beam data acquisition was very fast. For 12 cones and 12 IRIS field sizes, all the required scanning data can be obtained in less than one day.

\section{Additional Information}

\section{Disclosures}

Human subjects: All authors have confirmed that this study did not involve human participants or tissue. Animal subjects: All authors have confirmed that this study did not involve animal subjects or tissue. Conflicts of interest: In compliance with the ICMJE uniform disclosure form, all authors declare the following: Payment/services info: All authors have declared that no financial support was received from any organization for the submitted work. Financial relationships: All authors have declared that they have no financial relationships at present or within the previous three years with any organizations that might have an interest in the submitted work. Other relationships: All authors have declared that there are no other relationships or activities that could appear to have influenced the submitted work.

\section{References}

1. Accuray Physics Essentials Guide. Sunnyvale; 2005.

2. Bedford JL, Hansen VN, Webb S: The derivation of tissue-maximum ratio from percentage depth dose requires peak scatter factor to be considered a function of source-to-surface distance. Br J Radiol. 1998, 71: 876-881.

3. Reinstein LE, McShan DL: Computer-controlled direct TMR measurement. Med Phys. 1982, 9:917-919. 